\title{
닌
}

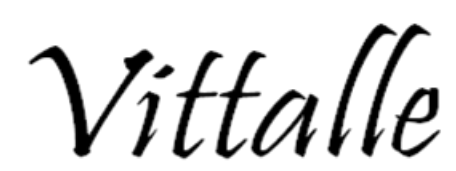

FURG

ISSN $2177-7853$

\section{Medicamentos para o tratamento da dor crônica: uma questão de acesso?}

\author{
Maria Cecília Carvalho Caetano ${ }^{a}$, Juliana Apolônio Martins ${ }^{\mathrm{a}}$, Thais Lorenna Souza Sales ${ }^{\mathrm{a}}$, \\ Alexandre Ernesto Silva ${ }^{a}$, Cristina Sanches ${ }^{\text {a* }}$
}

${ }^{a}$ Universidade Federal de São João Del Rei- Campus Centro Oeste Dona Lindu, Rua Sebastião Gonçalves Coelho, ${ }^{\circ}$ 400, Bloco E, gabinete 303.2, Bairro Chanadour, Divinópolis - MG, CEP 35.501-296.

Histórico do Artigo
Recebido em: 19/12/2016
Aceito em: 01/03/2017
Palavras-chave
Dor crônica
Assistência farmacêutica
Acesso aos Serviços de
Saúde

Histórico do Artigo

Recebido em: 19/12/2016

Palavras-chave

Dor crônica

Assistência farmacêutica

Saúde

Keywords

Chronic pain

Pharmaceutic Services

Health Services

Accessibility

*Autor correspondente: csanches@ufsj.edu.br

(C. Sanches)

\section{RESUMO}

A Organização Mundial da Saúde (OMS) apresenta como um pilar de sua política pública para o desenvolvimento do cuidado paliativo a disponibilidade do medicamento para o tratamento da dor crônica. No Brasil, a Portaria $\mathrm{n}^{\mathrm{o}} 1083 / 2012$ visa garantir este direito. O presente relato objetiva descrever e discutir as formas de acesso aos medicamentos em pacientes em cuidados paliativos e dor crônica, em três unidades: Secretaria de Estado de Saúde (SES), Unidade de Alta Complexidade em Oncologia (UNACON) e uma Organização Não Governamental (ONG). O processo para obtenção do medicamento via SES não é realizado devido à falta de orientação da equipe médica e disponibilidade de documentos para abertura do processo administrativo. Conseqüentemente, a ONG se responsabilizou pela aquisição e distribuição dos mesmos para os pacientes encaminhados do UNACON. Conclui-se que ainda não conseguimos garantir o acesso ao medicamento para pacientes em cuidados paliativos, necessitando de conscientização do prescritor e desburocratização do processo de dispensação.

Drugs for chronic pain treatment: an access issue?

ABSTRACT - The World Health Organization (WHO) presents as a pillar of his public policy for the development of palliative care, the drug availability for chronic pain treatment. In Brazil, Decree $\mathrm{n}^{\circ} 1083$ / 2012 aims to ensure this right. Thus, this report aims to discuss ways of drug access for patients in palliative care and chronic pain in three units: State Health Secretariat (SHS), High Complexity in Oncology Unit (UNACON) and an Non-Governmental Organization (NGO). The process for obtaining the drug via SHS is not carried out due to lack of guidance of the medical staff and availability of documents for opening of administrative proceedings. Consequently, the NGO was responsible for the acquisition and distribution of the same to patients referred from UNACON. It is concluded that even we cannot guarantee access to medication for patients in palliative care, requiring awareness of prescribers and bureaucracy of the dispensing process.

\section{Introdução}

As neoplasias acometeram mundialmente cerca de 17 milhões de pessoas nas últimas três décadas, sendo frequentemente acompanhada pela dor crônica. Com o uso de medicamentos é possível aliviar o sofrimento desses pacientes em até $90 \%$ dos casos (1). Desta forma, critérios para uma farmacoterapia racional foram criados pela Organização Mundial de Saúde (OMS) visando o desenvolvimento de políticas públicas para o bem estar do paciente que, além do diagnóstico e tratamento adequado, garantam a disponibilidade e o acesso ao tratamento medicamentoso (1). No entanto, apesar de todos os esforços, o acesso ao medicamento é insuficiente forçando os pacientes a co-existirem com a dor (2). 
No Brasil, medicamentos para o tratamento da dor crônica são classificados como pertencentes ao componente especializado da assistência farmacêutica; uma estratégia desenvolvida para garantir o acesso através de protocolos clínicos estruturados no âmbito do Sistema Único de Saúde (SUS) $(3,4)$. Para o recebimento desses os pacientes precisam estar dentro dos critérios de inclusão estabelecidos no Protocolo Clinico e Diretrizes Terapêutica da Dor Crônica estabelecido pela Portaria nº1083/2012 (4). Apesar destes esforços, verificase ainda que o acesso a medicamentos no país ainda é deficiente e a falta de documentação necessária para atender aos protocolos é o principal fator para o não fornecimento destes medicamentos $(5,6)$.

Adicionalmente, é imprescindível garantir que os direitos a saúde não serão violados, contrariando o Art. 196 da constituição de 1988 no que diz "a saúde é direito de todos e dever do estado" (7,8). Garantir o acesso de pacientes que sofrem com dor crônica aos medicamentos é de total importância para proporcionar qualidade de vida aos seus dias, sendo necessário eliminar os entraves que bloqueiam o acesso a esses medicamentos (9).

$\mathrm{O}$ presente relato surgiu da experiência do acompanhamento de professores e alunos dos cursos de graduação em Enfermagem, Farmácia e Medicina em um Projeto de Extensão denominado "Bem Cuidar". Esse Projeto foi criado em 2013, com o objetivo de melhorar a qualidade de vida de pacientes portadores de câncer, em cuidados paliativos e dor crônica, por meio de capacitação/educação para o cuidado e autocuidado em domicílio; sendo sua finalidade principal promover uma alta segura e capaz de gerar a continuidade dos cuidados no contexto domiciliar.

Durante a fase de implantação do projeto verificou-se a necessidade de estabelecimento de um fluxo de atendimento a estes pacientes em todas as especialidades envolvidas, para que os pacientes fossem direcionados. Para os discentes do curso de Farmácia o desafio era garantir alta hospitalar orientada quanto ao uso adequado dos medicamentos e recebimento destes ainda no ambiente hospitalar antes mesmo da alta.

Porém, observaram-se além da constatação da inexistência de um fluxo de atendimento aos pacientes acompanhados pelo Projeto, diversas tentativas de estabelecimento deste fluxo para garantir o acesso aos medicamentos do componente especializado da assistência farmacêutica foram realizadas, o que motivou o presente relato. Objetiva-se, então, descrever e discutir as formas de acesso aos medicamentos em pacientes em cuidados paliativos e dor crônica, em um município de médio porte do Centro Oeste Mineiro.

\section{Metodologia}

Trata-se de um estudo do tipo relato de experiência, gerado a partir das dificuldades de acesso aos medicamentos, para pacientes sem perspectivas terapêuticas de cura, geradas a partir do projeto de extensão "Bem Cuidar", proveniente de uma Universidade Federal estabelecida em uma cidade de médio porte do centro oeste mineiro.

As atividades foram desenvolvidas em três unidades:

(1) Organização Não Governamental (ONG) de suporte a pacientes com câncer - é uma entidade sem fins lucrativos, políticos ou religiosos, fundada em 21 de março de 1995. Atende uma população estimada em um milhão e 300 mil pessoas em cerca de 86 cidades no estado de Minas Gerais.

(2) Unidade de Alta Complexidade em Oncologia (UNACON) - presta assistência especializada de alta complexidade para o diagnóstico definitivo e tratamento dos cânceres mais prevalentes na região.

(3) Secretaria de Estado de Saúde (SES), Regional de Saúde - órgão governamental que atende 54 municípios mineiros. Realiza a distribuição e dispensação dos 
medicamentos do componente Especializado da Assistência farmacêutica, entre eles, os constantes na Portaria $n^{\circ}$ 1083/2012 para o tratamento da dor crônica.

Para o estabelecimento do fluxo de atendimento três etapas foram necessárias:

(1) "reconhecimento do cenário" - foram realizadas reuniões com as três entidades envolvidas no processo, com intermediação dos participantes do projeto "Bem Cuidar", visando o estabelecimento de um fluxo de atendimento.

(2) "identificação dos problemas" - os entraves foram levantados e novas rodas de conversas foram realizadas.

(3) "proposta de intervenções" - as propostas de intervenção foram apresentadas.

\section{Resultados e discussão}

Inicialmente foram realizadas três reuniões, sendo a primeira entre os membros do projeto "Bem Cuidar" e a SES para a busca de informações e fluxos pré-existentes sobre a autorização de procedimento de alta complexidade (APAC) - procedimento necessário para cadastramento do paciente. Dessa forma, somente após a coleta de todos os documentos para a abertura do processo poderia ser realizada a dispensação do medicamento.

A segunda reunião aconteceu entre os membros do projeto "Bem Cuidar", a ONG e a Secretaria Municipal de Saúde, ficando acordado que tanto o processo quanto a retirada dos medicamentos seriam realizados por representante legal da ONG em horário alternativo de atendimento aos pacientes.

A terceira reunião aconteceu entre os membros do projeto "Bem Cuidar", a ONG e UNACON, para a apresentação da proposta e estabelecimento do novo fluxo. Ficou estabelecido que os alunos do curso de farmácia identificariam os pacientes que teriam alta hospitalar e providenciariam a documentação necessária para a abertura da APAC. Com o estabelecimento de um novo fluxo, o paciente em alta hospitalar, teria acesso ao medicamento fora do âmbito hospitalar.

$\mathrm{O}$ primeiro problema observado foi à resistência dos prescritores do UNACON em confecção a documentação para a abertura da APAC conforme Portaria n ${ }^{\circ} 1083 / 2012$. A primeira solução proposta foi o preenchimento dos documentos via internet.

Outra situação observada foi a falta de "notificação de receita A1" (receituário amarelo), como estabelecido na portaria $n^{\circ} 344 / 1998$ (10). Todos os prescritores necessitavam de talonário amarelo, porém os mesmos não eram fornecidos pela instituição. Na tentativa de solucionar essa situação e obter maiores informações, uma nova reunião com a SES foi realizada, desta vez, com o setor responsável pela liberação dos talonários. Foi repassado pela instituição que o receituário amarelo é gratuitamente distribuído via SES através de talonários que contem 20 folhas cada e que um talonário por mês seria dispensado em nome de cada prescritor. Para isto, os prescritores deveriam ser individualmente cadastrados, através de formulário específico na SES. Questionou-se então a possibilidade dos talonários serem dispensados ao UNACON, em nome do Diretor Técnico da instituição. No entanto, a representante legal da SES evidenciou que número superior a um talonário só poderia ser dispensado através da apresentação de uma lista prévia com todos os nomes dos pacientes que fariam uso dos medicamentos para dor crônica no mês posterior à liberação. Esse requisito imposto seria impossível de ser acatado uma vez que o UNACON atende milhares de pacientes mensalmente, sendo muitos destes pacientes sem perspectiva de cura, em final de vida e com fluxo constante de internação e re-internação. Diante da não solução do problema sugeriu-se a dispensa de apresentação da receita amarela, com base na RDC $\mathrm{n}^{\circ}$ 202 de 18 de julho de 2002, no que diz: "a Notificação de Receita " $A$ " não será exigida para dispensação de medicamentos à base das substâncias morfina, medatona e codeína, ou 
de seus sais, a pacientes em tratamento ambulatorial, cadastrados no Programa Nacional de Assistência à Dor e Cuidados Paliativos, do Sistema Único de Saúde” (11). Entretanto, funcionários da SES não tinham conhecimento referente à Resolução, relatando ser uma prática ilegal e alegando que a portaria $344 / 98$ prevaleceria. Os autores buscaram ainda informações sobre a possibilidade de cadastro dos pacientes no "Programa Nacional de Assistência à Dor e Cuidados Paliativos", sem sucesso.

$\mathrm{Na}$ presença de todos esses impasses foi necessária outra reunião entre os membros do projeto "Bem Cuidar", a ONG e a diretoria do UNACON para expor as dificuldades quanto a notificação de receituário amarelo. Dessa forma, ficou estabelecido que o UNACON se responsabilizaria pela solicitação de talonários. No entanto, até o presente momento os talionários não haviam sido disponibilizados pelo UNACOM. A Tabela 1 apresenta os entraves encontrados nos diferentes níveis de atendimento ao paciente sem perspectivas terapêuticas de cura.

Tabela 1. Dificuldades encontradas nos diferentes níveis de atendimento ao pacientes sem perspectivas terapeuticas de cura provenientes de um municipio de medio porte mineiro.

\begin{tabular}{ccccc}
\hline Origem & Prescritor & UNACON & SES & ONG \\
\hline Dificuldade & $\begin{array}{c}\text { Preenchimento dos } \\
\text { documentos exigidos para } \\
\text { abertura de APAC }\end{array}$ & $\begin{array}{c}\text { Ausencia de } \\
\text { Talonário } \\
\text { Amarelo }\end{array}$ & $\begin{array}{c}\text { Controle Rigoroso } \\
\text { para liberacao de } \\
\text { Talonário Amarelo }\end{array}$ & $\begin{array}{c}\text { Dispensaçao de } \\
\text { opióides sem }\end{array}$ \\
$\begin{array}{c}\text { Solução } \\
\text { apresentada }\end{array}$ & $\begin{array}{c}\text { Preenchínento Amarelo } \\
\text { Formulário Eletrônico }\end{array}$ & $\begin{array}{c}\text { Solicitar } \\
\text { Talonário }\end{array}$ & $\begin{array}{c}\text { Seguir a RDC 202 } \\
\text { de 18 de junho de } \\
2002\end{array}$ & $\begin{array}{c}\text { Dispensar apenas } \\
\text { com a presença do } \\
\text { receituário }\end{array}$ \\
$\begin{array}{c}\text { Resultado da } \\
\text { Intervenção }\end{array}$ & Não aceito & Não realizado & Não aceito & $\begin{array}{c}\text { Necessidade de } \\
\text { reestruturação para } \\
\text { aceitação }\end{array}$ \\
\hline
\end{tabular}

APAC: autorizaçao para procedimento de alta complexidade.

Diante das fracassadas tentativas de se estabelecer um novo fluxo de atendimento via SES, verificou-se que o acesso dos pacientes oncológicos aos medicamentos para dor não é garantido devido, principalmente, a entraves burocráticos (Figura 1). Consequentemente, historicamente, a ONG se responsabilizou pela aquisição e distribuição dos medicamentos para os pacientes oncológicos que eram encaminhados do UNACON, na tentativa de atender ao paciente que, de outra forma, não receberia o medicamento. Esta dispensação era realizada por um técnico de enfermagem, sem a exigência de Notificação de Receita (receituário amarelo). É importante destacar que a ONG necessita de doações para o seu funcionamento, sendo que a redução dos gastos com aquisição de medicamentos poderia ser repassada para outras atividades ou melhoria da ONG. Além disto, tornou-se uma atividade cômoda para os prescritores da UNACON, que não necessitavam preencher os documentos necessários para a abertura da APAC. A partir de projeto a ONG se adequou aos requisitos legais: 1 - criando uma Farmácia de dispensação; 2 - contratando farmacêutico responsável técnico; 3 - exigindo além da receita, Notificação de Receita "A"; 4 - realizando dispensação orientada quanto ao uso racional dos medicamentos utilizados por estes pacientes.

O fluxograma 1 apresenta as possibilidades e caminhos para retirada dos medicamentos para o tratamento da dor crônica pelos pacientes sem perspectivas terapêuticas de cura, que pode retirá-lo na SES, na ONG ou ficar sem o medicamento pertencente ao componente especializado da Assistência Farmacêutica. Além deste fluxo, caso o paciente necessite de medicamentos do componente básico, como antieméticos, analgésicos, entre outros, deverá procurar a atenção primária do município. 


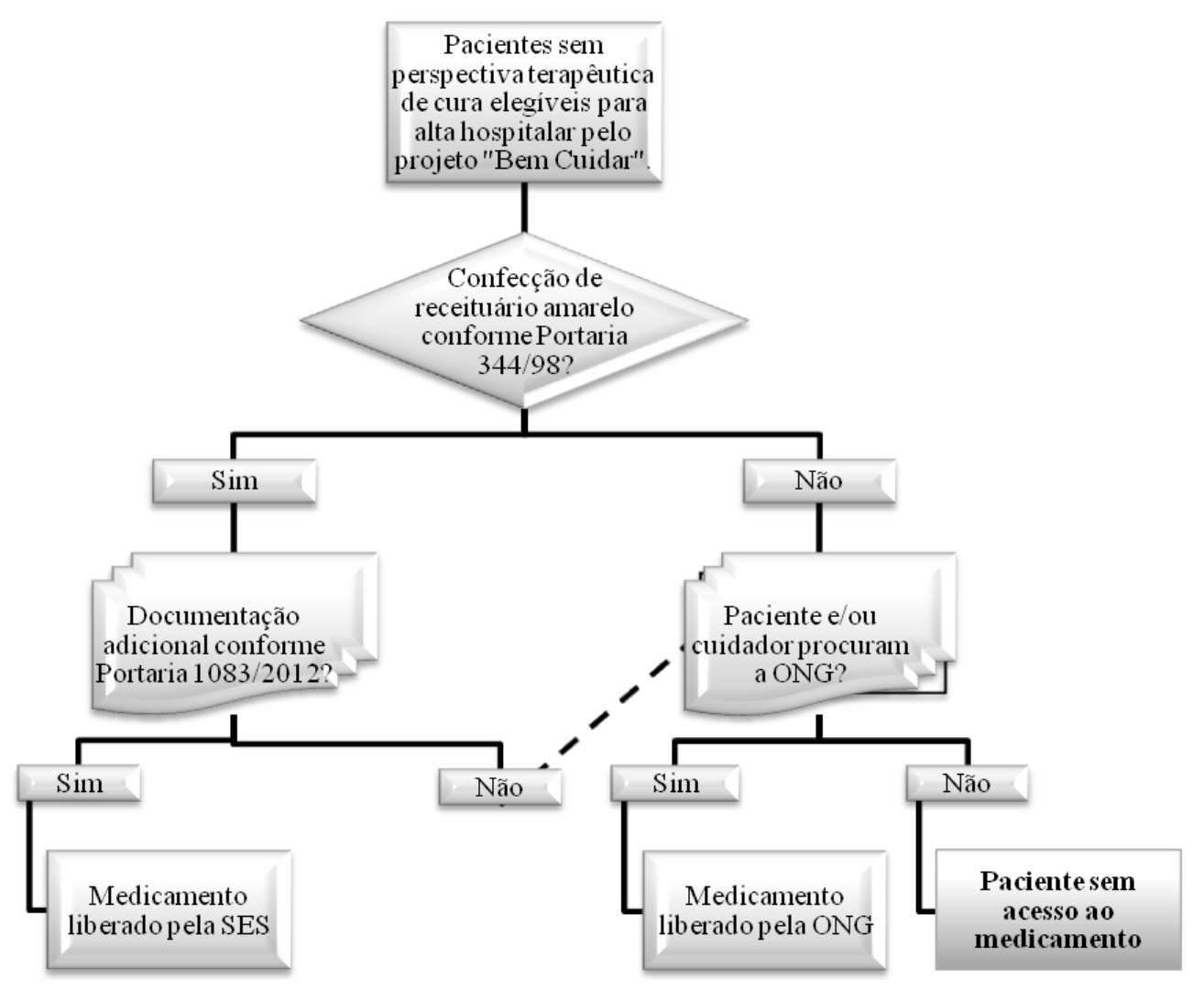

Figura 1. Fluxos de dispensação de medicamentos contidos na Diretriz Clinica de Tratamento da Dor crônica $^{4}$, na presença e ausencia de receituario amarelo. SES - Secretaria de Estado de Saúde; ONG Organização Não Governamental.

Apesar dos esforços e discussões, o acesso aos medicamentos ainda encontra barreiras que impedem o cumprimento das diretrizes do SUS. ${ }^{3}$ A dificuldade de acesso a medicamentos não é uma realidade apenas dos pacientes sem perspectivas terapêuticas de cura. Boing e colaboradores (2013), a partir de uma amostra domiciliar de 19.427 indivíduos verificaram uma prevalência de acesso a medicamentos de $45,3 \%$. Tanto a burocratização do processo de liberação do medicamento quanto a falta do trabalho em equipe em prol do paciente são apontados como fatores importantes que dificultam ou impedem o acesso aos medicamentos para dor crônica.

Dellamora et al. (6) ressalta que os profissionais envolvidos nesse processo exibem total falta de interesse, além de acharem que com a elaboração dos documentos poderiam sobrecarregar ainda mais suas atividades. A extrema burocratização dos protocolos leva ao não preenchimento dos formulários e notificação de receita por muitos profissionais, e conforme observado no presente relato, o mesmo também é observado em pacientes pertencentes ao protocolo de tratamento de dor crônica e em final de vida ${ }^{6}$. Todavia essa barreira pode ser contornada pela mudança quanto aos preenchimentos de toda documentação, desburocratizando o processo a fim de garantir o acesso. As mudanças dos protocolos podem ajudar na melhoria da assistência dos profissionais da saúde ao paciente (12).

Outro gargalo que atrapalha e dificulta o acesso destes pacientes aos medicamentos de acordo com o estudo de Dellamora et al (6), é a falta de trabalho em equipe, pois para o sucesso do mesmo é imprescindível a participação de mais de um profissional de saúde. A 
união de todos os profissionais de uma instituição é fundamental para contornar dificuldades presentes.

Quando se tem metas e objetivos em comum entre os profissionais, obter sucesso para a resolução do problema é muito mais simples (13). O impasse entre as profissões dificulta os esclarecimentos de dúvidas e preenchimento dos documentos para que o paciente tenha acesso à farmacoterapia, impedindo a melhora nos serviços de saúde $(6,14)$. Dessa forma, no presente relato um melhor relacionamento entre os profissionais nos diferentes níveis de atuação e em instituições diferentes poderia modificar o cenário, a fim de que cada profissional conheça de fato o seu papel visando garantir a disponibilidade do medicamento ao paciente com dor crônica.

Em meio a esses impasses, o paciente se vê sem a medicação vivenciando dias marcados por sofrimento constante (15). A falta do medicamento é acompanhado pela condição clínica do paciente, além de afetar os membros da família, que se sentem impotentes frente às limitações para sanar as dores (15). Todo tratamento farmacológico é essencial para o paciente e dever do Estado. No entanto, apesar de estar disponível no SUS através do componente Especializado da Assistência Farmacêutica, infelizmente, o paciente não tem acesso ao medicamento (4).

O acesso é um problema de saúde pública que atinge o território nacional de forma desigual (16,18). Paniz et al. (17,21) observa diferenças no acesso entre as regiões do Brasil, as condições socioeconômicas, raça e escolaridade. Lima et al. (19) também relata diferenças no acesso entre unidades de um mesmo município. A presença de falhas na gestão agrava mais ainda essa situação, o que leva a diferenças e dificuldades em todo o ciclo da assistência farmacêutica. Finalmente, soma-se a estes fatores, a pouca ou nenhuma comunicação entre os órgãos e entidades públicas e privadas, a falta de informação para os usuários e a forma de dispensação de medicamentos seguindo a lógica do financiamento (básico, estratégico e especializado) (22-23).

\section{Considerações finais}

Diante da situação, o novo fluxo de alta orientada e dispensação de medicamentos para o tratamento da dor crônica em pacientes sem possibilidades terapêuticas de cura não foi implantado por entraves burocráticos presentes nos protocolos. Dessa forma, a sociedade tem feito seu papel, disponibilizando estes medicamentos através da ONG. Porém, o impacto financeiro para a organização é elevado e, uma vez que sua sobrevivência é dependente de filantropia, os recursos destinados à aquisição de medicamentos poderiam ser revertidos em outros ganhos para estes pacientes. Sendo assim, maiores discussões são necessárias visando garantir o acesso ao medicamento, orientação ao paciente, conscientização do prescritor e desburocratização do processo de dispensação. O olhar deve ser sempre voltado e repensado para o paciente.

Agradecimentos: Os autores são gratos a Simone Melo Souza, Camila Oliveira Ribeiro e Jessica Cunha Trindade pelo apoio no desenvolvimento deste trabalho. À Associação de Combate ao Câncer do Centro Oeste Mineiro (ACCCOM) pelo apoio.

Conflito de interesses: Os autores declaram não haver conflito de interesses

\section{Referências}

1-Minson FP. et al. II Consenso Nacional de Dor Oncológica. 1ª edição. São Paulo: Editora Moreira Junior; 2011. 
2- Pimenta CAM, Koizumi MS, Teixeira MJ. Dor no doente com câncer: características e controle. Ver Bras de Cancerologia [periódico na Internet]. 1997 Jan-Mar [acessado 2015 setembro]. Disponível em: http://www.inca.gov.br/rbc/n_43/v01/artigo2_completo.html.

3-Ministério da Saúde (MS). Componente especializado da Assistência Farmacêutica: Inovação para garantia do acesso a medicamentos no SUS. Brasília; 2014.

4- Brasil. Portaria ${ }^{\circ} 1083$ de 2 de Outubro de 2012. Protocolo Clínico e diretrizes terapêuticas dor crônica. Diário Oficial da União 2012; 03 out.

5- Vieira FS. Assistência farmacêutica no sistema público de saúde no Brasil. Rev Pan Salud Publica 2010; 27(2): 149-56.

6- Dellamora ECL, Caetano R, Castro CGSO. Dispensação de medicamentos do componente especializado em polos no estado do Rio de Janeiro. Cien Saúde Colet 2012; 17 (9): 2387- 2396.

7- Schwartz TD, Ferreira JTB, Maciel ELN, Lima RCDL. Estratégia saúde da família: avaliando o acesso ao SUS a partir da percepção dos usuários da Unidade de Saúde de Resistência, na região de São Pedro, no município de Vitória (ES). Cien Saúde Colet 2010; 15 (4): 2145-2154.

8- Brasil. Constituição (1988). Constituição da República Federativa do Brasil. Brasília, DF: Senado Federal: 1988.

9- Paniz VMV. Acesso a medicamentos em população assistida por diferentes modelos de atenção básica nas regiões Sul e Nordeste do Brasil. [tese]. Pelotas: Universidade Federal de Pelotas; 2009.

10- Brasil. Portaria $\mathrm{n}^{\circ} 344$ de 12 de Maio de 1998. Regulamento Técnico sobre substancias e medicamentos sujeitos a controle especial. Diário Oficial da União, 1988; 12 maio.

11-BRASIL. Rdc n 202 de 18 de Julho de 2002. Determina que a Notificação de Receita "A" não será exigida para dispensação de medicamentos à base das substâncias morfina, medatona

e codeína, ou de seus sais, a pacientes em tratamento ambulatorial, cadastrados no Programa Nacional de Assistência à Dor e Cuidados Paliativos, do Sistema Único de Saúde e dá outras providências. Diário Oficial da União 2002; 18 jul.

12- Honório RPP, Caetano JA. Elaboração de um protocolo de assistência de enfermagem ao paciente hematológico: relato de experiência [serial on the internet] 2009; 11(1): [188-93]. Available from: http://www.fen.ufg.br/revista/v11/n1/v11n1a24.htm

13- Grego RM. Ensinando a Administração em Enfermagem através da Educação em Saúde. Rev Bras Enferm 2004; 57(4):504-7.

14. Peduzzi M. Equipe multiprofissional de saúde: conceito e tipologia. Rev Saúde Pública 2001; 35(1):103-9.

15- Miceli AVP. Dor crônica e subjetividade em oncologia. Rev Bras Cancerologia 2002; 48(3): $363-37$.

16- Louvison MCP, Lebrão ML, Duarte YAO, Santos JLF, Malik AM, Almeida ES. Desigualdades no uso e acesso aos serviços de saúde entre idosos do município de São Paulo. Rev Saúde Pública 2008; 42(4):733-40.

17- Paniz VMV, Fassa ACG, Facchini LA, Bertoldi AD, Piccini RX, Tomasi E, Thumé E, Silveira DS, Siqueira FV, Rodrigues MA. Access to continuous-use medication among adults

and the elderly in South and Northeast Brazil. Cad. Saúde Pública 2008; 24(2):267-280.

18- Chieffi AL, Barata RB. Judicialização da política pública de assistência. Cad Saúde Pública, 2009; 25(8):1839-1849.

19- Lima MADS, Ramos DD, Rosa RB, Nauderer TM, Davis R. Acesso e acolhimento em unidades de saúde na visão dos usuários. Acta Paul Enferm 2007;20(1):12-7.

20 - Boing AC, Bertoldi AD, Boing AF , Bastos JL, Peres KG. Acesso a medicamentos no setor público: análise de usuários do Sistema Único de Saúde no Brasil. Cad. Saúde Pública, Rio de Janeiro, 29(4):691-701, abr, 2013.

21 - Paniz Vera Maria Vieira, Fassa Anaclaudia Gastal, Facchini Luiz Augusto, Bertoldi Andréa Dâmaso, Piccini Roberto Xavier, Tomasi Elaine et al . Acesso a medicamentos de uso contínuo em adultos e idosos nas regiões Sul e Nordeste do Brasil. Cad. Saúde Pública [Internet]. 2008 Feb [cited 2017 Feb 15] ;24( 2 ): 267-280.

22 - Baldoni AO, Dewulf NLS, Santos V, Reis TM, Ayres LR, Pereira LRL. Dificuldades de acesso aos serviços farmacêuticos pelos idosos. Rev Ciênc Farm Básica Apl., 2014;35(4):615-621.

23 - Vieira Fabiola Sulpino. Pharmaceutical assistance in the Brazilian public health care system. Rev Panam Salud Publica [Internet]. 2010 Feb [cited 2017 Feb 15] ; 27( 2 ): 149-156. 